\title{
Alloimmune T cells in transplantation
}

\author{
Susan DeWolf and Megan Sykes \\ Columbia Center for Translational Immunology, Department of Medicine, Columbia University, New York, New York, USA.
}

\begin{abstract}
Alloimmune T cells are central mediators of rejection and graft-versus-host disease in both solid organ and hematopoietic stem cell transplantation. Unique among immune responses in terms of its strength and diversity, the T cell alloresponse reflects extensive genetic polymorphisms between allogeneic donors and recipients, most prominently within the major histocompatibility complex (MHC), which encodes human leukocyte antigens (HLAs) in humans. The repertoire of alloreactive T cell clones is distinct for every donor-recipient pair and includes potentially thousands of unique HLA/peptide specificities. The extraordinary magnitude of the primary alloresponse and diversity of the T cell population mediating it have presented technical challenges to its study in humans. High-throughput T cell receptor sequencing approaches have opened up new possibilities for tackling many fundamental questions about this important immunologic phenomenon.
\end{abstract}

\section{Introduction}

Transplantation provides lifesaving organs to patients with endstage organ failure and lifesaving hematopoietic cell grafts to individuals with malignant or nonmalignant hematologic disorders. The success of these transplants depends on potent nonspecific immunosuppressive therapy to prevent graft rejection and graft-versushost disease (GVHD). Alloimmune T cells are the backbone of the human adaptive immune response to transplants of organs, cells, and tissues from other humans, which are referred to as allogeneic. This alloimmune response is the central immune response in solid organ transplantation and hematopoietic stem cell transplantation (HSCT), in both host-versus-graft and graft-versus-host responses. Fundamental questions about the alloimmune response have challenged immunologists since research in transplantation began. The response to allogeneic major histocompatibility complex (MHC), or, specifically in humans, human leukocyte antigens (HLAs), differs from responses to more classical antigens, such as those derived from pathogens or self, because of its extraordinary strength and the apparent size and diversity of the alloreactive repertoire. The alloimmune $\mathrm{T}$ cell repertoire against a given allogeneic $\mathrm{MHC}$ haplotype has been estimated to constitute $1 \%-10 \%$ of the entire T cell population. The studies leading to these widely cited values usually relied on in vitro or in vivo functional assays (1-12). While such studies, along with understanding of mechanisms of allorecognition, suggested that the alloreactive repertoire was likely to be vast, methods of actually quantifying it were not available at the time. Here we review the immunology of the alloimmune $\mathrm{T}$ cell response in transplantation and discuss how emerging approaches based on $\mathrm{T}$ cell receptor (TCR) sequencing may provide new insights into this response.

\section{Types of allorecognition}

Allorecognition in vivo can be divided into three separate categories: direct, indirect, and semidirect pathways (refs. 13, 14, and Fig-

Conflict of interest: M. Sykes has submitted a Provisional Application for a US patent (14/892,512, 2016).

Reference information: / Clin Invest. 2017;127(7):2473-2481.

https://doi.org/10.1172/JCI90595. ure 1). T cells reacting directly to alloantigens presented by donor antigen-presenting cells (APCs) mediate the "direct" alloresponse. This response is classically associated with acute rejection (15), is known for its unique strength, and is expected to be diverse. Its potency is responsible for the strength of the primary alloresponse detected by mixed lymphocyte and cell-mediated lympholysis reactions without prior priming in vivo or in vitro. The indirect alloresponse, in contrast, resembles more typical immune responses in which T cells recognize self-APCs presenting peptides on self-HLA molecules; however, the peptide originates from donor MHC antigens or other polymorphic proteins. Chronic rejection is thought to include a major role for indirect allorecognition, as donor APCs in the graft are replaced by those of the recipient over time. Indirect allorecognition can, for example, induce graft vasculopathy in an experimental model (16). Moreover, alloantibodies are strongly associated with chronic rejection, and their production is facilitated by cognate interactions between alloreactive B cells with immunoglobulin receptors that bind donor HLA molecules and internalize them, resulting in focused presentation to indirectly alloreactive $\mathrm{T}$ cells that recognize peptides from the same allogeneic HLA molecules and help antibody production by those B cells (17).

The clinical significance of the semidirect immune response is beginning to emerge. In semidirect allorecognition, intact allogeneic HLA/peptide complexes that have been transferred from donor cells to recipient cells, a process sometimes referred to as "cross-dressing," activate T cells (18). A recent study in rodents suggests that unexpectedly high numbers of recipient APCs acquire donor MHC molecules via microvesicles (exosomes) during the transplantation process, supporting a role for the semidirect pathway in rejection (19).

The human alloresponse measured in vitro involves CD4 and CD8 cells in both naive and memory T cell compartments $(20,21)$. Greater HLA mismatching would be expected to increase the diversity of the alloreactive repertoire, but studies directly addressing this question are lacking. Because memory T cells may not require costimulation for activation and persist at higher frequencies in the circulation than naive $\mathrm{T}$ cells, assays may be biased toward the detection of alloreactive memory $\mathrm{T}$ cells. However, while the role 
Direct pathway

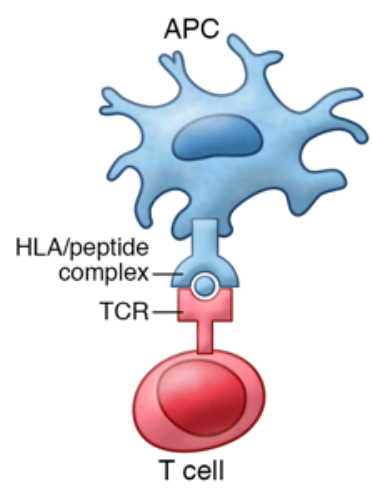

Donor Recipient

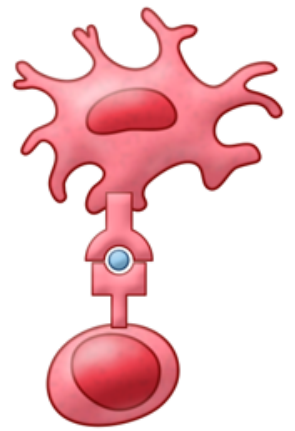

Semidirect pathway

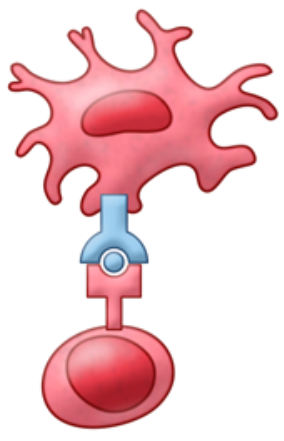

Figure 1. Pathways of allorecognition. Schematic illustration of the three major pathways of allorecognition: direct, indirect, and semidirect. In the direct pathway, donor antigen-presenting cells (APCs) interact directly with recipient $T$ cells. In indirect recognition, recipient APCs present processed donor allogeneic peptides to recipient $\mathrm{T}$ cells, similar to more typical immune responses. In the semidirect pathway, recipient APCs acquire donor HLA molecules that present peptides directly to recipient $\mathrm{T}$ cells. of cross-reactive memory $\mathrm{T}$ cells in mediating allograft rejection has been emphasized in animal studies (22-26), murine studies (27-29) have shown that effector memory CD4 cells have reduced ability to induce GVHD while maintaining graft-versus-leukemia effects. Additionally, recent clinical study results (30) are consistent with a role for naive $\mathrm{T}$ cells in inducing GVHD.

The relative contribution of CD4 and CD8 cells, direct and indirect pathways, and naive and memory $\mathrm{T}$ cells to an alloresponse is likely to depend on a number of variables, including the extent and class of HLA mismatching, infectious exposures, and many others, all of which contribute to the complexity of the human alloresponse.

\section{Remarkable strength of the alloresponse}

The exceptional potency of the alloresponse predominantly reflects the extraordinary polymorphism of the mammalian MHC, combined with the primary role of these molecules in presenting antigens to TCRs. MHC molecules, or, specifically in humans, HLAs, present antigens in the form of peptides that are seen by uniquely rearranged TCRs. The multiple "classical" HLA loci that can present peptide antigens (HLA-DR, -DQ, and -DP class II antigens and HLA-A and -B class I antigens), combined with this polymorphism, result in hundreds of alleles that permit robust individual immune responses to diverse pathogens and, on a population level, help to avoid species destruction by a particular pathogen (31).

While the extent of HLA matching clearly correlates with organ transplant outcomes (32-34), the vast majority of organ transplants are performed across HLA barriers. In contrast, until recently the need for close HLA matching was of primary importance in HSCT, as the risk of severe GVHD was very high when major HLA barriers were traversed (35). This situation has been somewhat alleviated by the use of HLA-mismatched cord blood transplantation and, more recently, by the use of post-transplant cyclophosphamide to reduce GVHD risk following HLA-mismatched transplantation $(36,37)$. Efforts are also ongoing to rationally identify low-risk "permissive" HLA mismatches based on which amino acid residues most affect peptide binding (38). While we emphasize alloreactivity against HLA polymorphisms in this Review because HLA barriers are typically traversed in solid organ transplantation, the hundreds of "minor" histocompatibility antigens resulting from presentation by shared HLA alleles of polymorphic peptides from non-MHC proteins are collectively significant, resulting in GVHD in $30 \%-50 \%$ of HLA-identical HSCT recipients despite immunoprophylaxis (39).

\section{Explaining the high frequency of $\mathrm{MHC}$ - alloreactive $T$ cells}

The ability to detect primary anti-MHC alloresponses in vitro and the potent rejection responses induced by allogeneic MHC indicate that these are unusually powerful immune responses. Evidence suggests that this potency may arise from the combination of inherent MHC recognition by the TCR structure, positive selection by low-affinity interactions with self-MHC/peptide complexes, and the inherent cross-reactivity/flexibility of TCR interactions with $\mathrm{MHC} /$ peptide complexes. Indeed, many studies point toward a germline affinity of TCRs for MHC (40-43). The low level of anti-self-reactivity in the normal peripheral $\mathrm{T}$ cell repertoire is believed to be dependent on the panoply of self-MHC/peptide complexes that contribute to thymic deletion of T cells whose receptors bind with relatively high affinity to those complexes. There is no such negative selection for $\mathrm{T}$ cells recognizing allogeneic MHC/peptide complexes. Thus, the TCR repertoire that is positively selected for low affinity to self-MHC/peptide and, by cross-reaction, receives higher affinity signals from foreign peptides presented by self-MHC, also has the potential to interact with high affinity to allogeneic MHC/peptide complexes. While some studies support the notion that the allo-MHC molecule alone is a major target of allorecognition $(44,45)$, there is ample evidence that the combination of the allo-MHC and peptide underlies direct allorecognition (46-52), consistent with the notion of high-affinity cross-reactivity with allo-MHC/peptides of TCRs with low affinity for self-MHC/peptide. The ability of TCRs to recognize multiple different MHC/peptide combinations, or degeneracy, could explain a diverse, cross-reactive $\mathrm{T}$ cell alloresponse $(53,54)$. It has been suggested that alloreactive $\mathrm{T}$ cells may be able to bind different portions of allo-MHC than of self-MHC and this structural flexibility in TCR binding may be a major contributor to the alloreactive potential of both CD4 and CD8 T cells (55-58). Diverse 


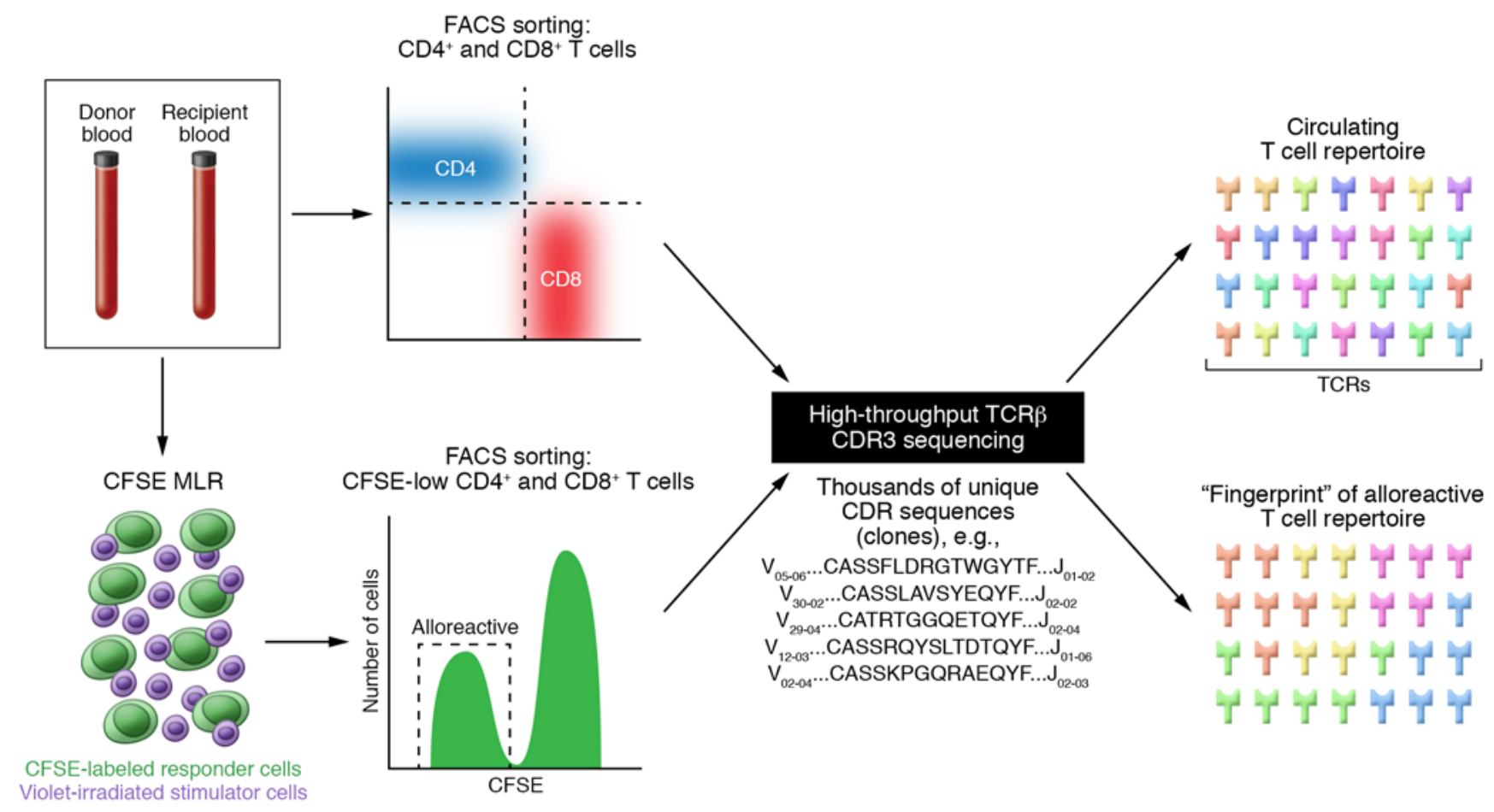

Figure 2. Defining a "fingerprint" of the alloresponse via TCR sequencing. From peripheral donor and recipient blood, unstimulated T cells and alloreactive T cells, isolated via FACS of CFSE-low cells in a recipient antidonor MLR, undergo high-throughput TCR $\beta$ CDR3 sequencing. Computational processing enables definition of a "fingerprint" of the alloreactive T cell population for any potential donor-recipient pair.

MHC/peptide complexes may have structural mimicry from the "point of view" of the TCR (49). Some evidence also suggests that alloreactive $\mathrm{T}$ cells are enriched for those expressing two distinct TCRs due to the presence of two different rearranged $\alpha$ chains, thereby increasing the potential for cross-reactivity $(59,60)$.

Cross-reactivity of pathogen-reactive TCRs is likely to explain the contribution of memory $\mathrm{T}$ cells to alloreactivity in recipients lacking prior exposure to alloantigens because of transfusion, pregnancy, or transplantation itself. Indeed, murine studies have demonstrated the ability of virus-reactive $\mathrm{T}$ cells to block tolerance induction due to broad-based cross-reactive alloimmunity $(25,26)$. EBV- and herpes simplex virus-specific clones have been shown to also react to allogeneic HLA in humans, particularly within the CD8 compartment (21, 61-65); it has even been suggested that nearly half of the TCRs specific to a given virus may also have alloreactivity (66). Furthermore, these viral peptide-specific TCRs may have "public" sequences shared between individuals with the same presenting HLA allele (67-70). Notably, cytokine production and other measures of $\mathrm{T}$ cell function are not always identical in response to viral antigen and cross-reactive HLA alloantigens (21), perhaps because of differing TCR affinities for different ligands. The notion that $\mathrm{T}$ cells that are cross-reactive to pathogens encountered during life are significant contributors to the alloresponse is consistent with the apparent importance of memory $\mathrm{T}$ cells in mediating allograft rejection (71-73). However, as discussed above, the apparent dominant role of naive $\mathrm{T}$ cells in $\operatorname{GVHD}(27,29,30)$ calls this notion into question, and because naive human $\mathrm{T}$ cells acquire the memory phenotype under lymphopenic conditions (74), studies showing a role for "memory" cells in rejection under such circumstances should be interpreted with caution. Thus, additional work is needed to fully understand the role of cross-reactive pathogen-specific $\mathrm{T}$ cell memory in the human alloresponse.

\section{Diversity of alloreactive TCRs}

$\mathrm{T}$ cell clones each have a unique nucleotide sequence encoding the TCR hypervariable region that determines antigen specificity. In $\alpha \beta$ $\mathrm{T}$ cells, a heterodimer results from the pairing of TCR $\alpha$ chains and $\beta$ chains (75). Though different portions of both the $\alpha$ and $\beta$ chains play integral roles in determining antigen specificity, it is the complementarity-determining 3 region (CDR3) (76) that undergoes somatic recombination of variable (V), diversity (D), and joining (J) genes, along with insertion of nontemplated nucleotides, which leads to the tremendous CDR3 diversity and thus TCR diversity of the millions of $\mathrm{T}$ cell clones in an individual $(77,78)$.

While most primary peptide antigen-specific immune responses reflect recognition by a small number of TCRs, early studies demonstrated remarkable diversity of $\mathrm{V} \beta$ usage contributing to the alloresponse against a single MHC allelic difference, suggesting that the overall alloreactive repertoire was broad $(79,80)$. Similarly, measurement of the distribution of lengths of TCR $\beta$ CDR3s, known as spectratyping, confirmed the broad repertoire of alloreactive TCRs (81).

\section{Methods for measuring the alloresponse}

Estimates of the size of the alloreactive $\mathrm{T}$ cell repertoire have, until recently, relied on functional assays, including bulk mixed lymphocyte reactions (MLRs) (82) and cell-mediated lympholysis (CML) assays, as well as more quantitative limiting diluting assays 
(LDAs). In MLR assays $(83,84)$, the donor or stimulator cells are irradiated (one-way MLR) and cocultured with recipient lymphocytes for 5-7 days, and responses to alloantigens are measured by $\mathrm{T}$ cell proliferation, either via incorporation of radioactive thymidine into the nucleic acids of dividing cells or using the dilution of intravital dyes such as CFSE and flow cytometry $(85,86)$. CML assays are similar to MLR assays, except the readout is killing of labeled donor target cells or cell surface expression of CD107a, reflecting granule exocytosis. In LDAs, precursor frequencies of antigen-specific cells are estimated via secretion of cytokines or cytotoxicity after activation in culture with progressively diluted concentrations of responder cells while the number of allogeneic stimulator cells remains constant; using a model based on the Poisson distribution, one can extrapolate the frequency of antigen-specific cells, including alloreactive cytotoxic and helper $\mathrm{T}$ cells. Finally, the enzyme-linked immunospot assay (ELISPOT) assay can be used to measure cytokine-producing cells responding to alloantigens, and rapid responses have been used to indicate the presence of allosensitized T cells $(87,88)$. While all of these assays provide valuable information about the function of alloimmune $\mathrm{T}$ cells, none of them actually identify alloreactive $\mathrm{T}$ cells independently of their function. MHC/peptide tetramers enable identification and isolation of distinct antigen-specific $\mathrm{T}$ cell populations for detailed functional and structural analysis (89), but the tremendous number of putative HLA/peptide combinations potentially recognized by alloreactive $\mathrm{T}$ cells precludes such an approach to studying the broad alloresponse.

Before the advent of high-throughput approaches, TCR clonotyping enabled identification and tracking of small numbers of alloreactive TCRs in transplant recipients. Michalek et al. identified a single allospecific clone in a donor antirecipient pretransplant MLR in a patient who had received a hematopoietic stem cell transplant from an allogeneic donor with one HLA-DRB1 mismatch (90). Tracking of this clone in the peripheral blood over time via PCR revealed an expansion in frequency at the time of GVHD and frequency reduction with steroid treatment. While the clone served as a marker, the studies of this clone did not capture events affecting the entire alloreactive repertoire.

In view of the limitations of the methods described above, we recently developed a high-throughput TCR sequencing-based approach for studying the human alloresponse. The emergence of high-throughput techniques for sequencing millions of TCR hypervariable regions in parallel (77) has made it possible to measure alloimmune $\mathrm{T}$ cells in a new way, permitting identification and tracking of thousands of alloreactive TCRs in the presence of any degree of HLA mismatching. Our approach to identifying and tracking a "fingerprint" of the donor-specific T cell repertoire involved combining the CFSE-MLR with high-throughput TCR sequencing (ref. 91 and Figure 2). The technique entails coculture of pretransplant recipient and irradiated donor mononuclear cells labeled with different fluorescent dyes for 6 days, followed by extraction of genomic DNA (gDNA) from sorted recipient CD4 and CD8 $\mathrm{T}$ cells that proliferate in response to donor antigens and thereby dilute their CFSE dye. This gDNA is then subjected to high-throughput TCR $\beta$ CDR3 sequencing along with gDNA extracted from unstimulated sorted pretransplant CD4 and CD8 $\mathrm{T}$ cells. Comparison of the pretransplant stimulated and unstim- ulated sequences permits designation of those TCRs whose frequencies increase markedly in the MLR as alloreactive, thereby defining a "fingerprint" of the recipient antidonor T cell repertoire that typically includes thousands of unique TCR $\beta$ CDR3 sequences. These clones can then be tracked in the peripheral circulation after transplant without the need for further functional assays or knowledge of alloreactive HLA/peptide specificities.

This TCR sequencing approach provided an unprecedented ability to determine the fate of donor-specific T cells in a cohort of tolerant combined kidney and bone marrow transplant (CKBMT) recipients (91). Expansion of circulating antidonor TCRs in nontolerant kidney transplant recipients demonstrated the biological relevance of the clones identified as donor-specific via this technique. By interrogating the post-transplant circulating T cell pool of TCRs, we obtained evidence that donor-reactive $\mathrm{T}$ cells were clonally reduced in the tolerant recipients and not in nontolerant recipients. While previous studies assessed general changes in TCR V $\beta$ usage and spectratypes over time after transplant $(92,93)$, this was the first study to specifically track particular TCRs that were identified as donor-reactive and compare their fate with that of those identified as non-donor-reactive. The latter provide an indication of overall $\mathrm{T}$ cell turnover resulting from induction and immunosuppressive therapy. Our observations led us to conclude that the presence of donor antigen during the period of lymphopenia following induction therapy drove the early expansion of donor-specific T cells (91). This finding is consistent with murine studies showing that rapid lymphopenia-driven proliferation is largely antigen-driven $(94,95)$.

This approach can also be used to gain a window into events in the allograft itself. TCR sequencing of post-transplant biopsies from tolerated allografts of CKBMT recipients revealed similar sequence dominance to that observed in the peripheral circulation at the same time, including a paucity of donor-reactive clones. In view of the absence of infiltrates observed histologically, these results suggest that the sequences obtained may have been from $\mathrm{T}$ cells in the kidney microvasculature (96).

We also applied this technique to sequential surveillance biopsy specimens in a cohort of intestinal transplant recipients and thereby gained new insights into human transplantation biology and mucosal immunology. Contrary to the dogma that $\mathrm{T}$ cell infiltrating rejecting allografts are largely bystanders (97), rejection episodes were associated with marked predominance of host-versus-graft-reactive (HVG-reactive) clones among TCR sequences identifiable as recipient in origin, demonstrating that bystander $\mathrm{T}$ cells are not a major component of $\mathrm{T}$ cell infiltrates during rejection. Since intestinal grafts come with large donor lymphoid loads and can be associated with GVHD, we identified the graft-versus-host $(\mathrm{GVH})$ alloreactive $\mathrm{T}$ cell fingerprint as well, using pretransplant donor lymphocytes stimulated with recipient APCs. Remarkably, the grafts demonstrated marked expansion of GVH-reactive T cells in association with early replacement of the major graft myeloid cell compartment by cells of recipient origin. These recipient myeloid cells likely served as APCs that expanded GVH-reactive T cells within the graft (98). These GVH-reactive T cells may have contributed to the peripheral blood $\mathrm{T}$ cell chimerism that was associated with reduced rejection rates in these patients, usually without GVHD (99). We also found that the balance of GVH and HVG clones 
within the graft after transplant correlated with rejection-related outcomes and that alloreactive $\mathrm{T}$ cell populations ultimately became a major component of the tissue-resident $\mathrm{T}$ cell repertoire in the gut, even extending to the native host intestine. Collectively, these studies, which combined the TCR tracking approach with multicolor flow cytometry, provided evidence that tissue-resident memory $\mathrm{T}$ cells transferred in the human intestinal allograft are derived from circulating donor central memory cells and that their repopulation after transplantation is largely driven by antigen reactivity, including infiltrating HVG clones and expanding tissue-resident GVH-reactive T cells (98). The persistence of expanded HVG clones as tissue-resident memory $\mathrm{T}$ cells in the graft may pose a constant risk of rejection and may help to explain the high rejection rates of grafts containing mucosal tissue, such as the intestine and the lung.

The study discussed in the preceding paragraph illustrates the power of the alloreactive TCR tracking approach to help elucidate events within the tissues following transplantation and further validates the biological significance of TCR sequences identified as alloreactive by this method. A similar approach could be useful in the setting of HSCT, where the enumeration of GVH-reactive clones within the GVHD target tissues could, perhaps in conjunction with analyses of such clones in the circulation, provide a new kind of alloantigen-specific diagnostic and prognostic information.

While there are several strategies for identifying $\mathrm{T}$ cells reactive to alloantigens, the CFSE-MLR used in our studies lends itself well to sequencing analysis, as FACS can be used to isolate alloreactive $\mathrm{T}$ cell populations for sequencing $(91,100)$. Upregulation of CD154 in the presence of anti-CD40 antibody during coculture is another potential technique, especially for CD4 cells, along with other methods to identify allospecific TCRs in MLRs (101-103).

\section{Technical considerations}

The studies discussed above provide strong evidence that clones identified as alloreactive via the MLR/TCR sequencing approach are biologically significant in patients. The definition of alloreactive clones has been based on consideration of several factors. The first is that highly abundant nonalloreactive clones resulting from clonal expansions in response to pathogens encountered in vivo may proliferate as bystanders in the MLR and thus be included in the CFSE-low population. For this reason, we compare the sequencing results from the CFSE-low stimulated cells with those of the unstimulated circulating cell populations at the same time, permitting the addition of a "fold-expansion" (in CFSE-low versus unstimulated populations) criterion for designation of CFSElow cells as alloreactive. Notably, the significance of the relative decline in donor-specific clones in tolerant a patient increased as we increased our fold-expansion criteria for defining alloreactive clones (91), suggesting that the more strongly alloreactive clones were more likely to be deleted than those with weaker reactivity. Furthermore, the chance of detecting clonal expansion of donor-reactive clones in nontolerant patients following transplant was not significantly altered by adjustment of the fold-expansion criterion for defining these clones (91).

In our view it is important to perform FACS on unstimulated and CFSE-low CD4 and CD8 T cell populations to allow separate analysis of their TCR repertoires, as the direction of changes in circulating alloreactive CD4 and CD8 clones during rejection may be different, as suggested in our early study (91). Furthermore, identification of the subset to which circulating or lymphoid tissue $\mathrm{T}$ cells belong before transplant provides additional information about clones identified in unsorted specimens from, for example, tissue biopsies $(96,98)$. However, because FACS is not without error, it is important to perform postsequencing data processing to eliminate ambiguous clones (those present at similar frequencies in sorted CD4 and CD8 populations) and to reassign mis-sorted clones (those that are clearly present at much greater frequency in one subset than the other).

One of the unexpected findings from our sequence-based analysis of alloreactive $\mathrm{T}$ cells was that, although such clones are numerous, they are generally present at low or undetectable frequency in the unstimulated circulating pool (our unpublished observations). Thus, the fold expansion of many alloreactive clones detected in MLR would be considered infinite, as these clones are not detected in the sequenced unstimulated population. In order to avoid including clones of this kind that may have expanded less than the requisite fold expansion to be defined as alloreactive, a minimum frequency threshold is included in the stimulated population based on the threshold for reliable detection of a clone in the unstimulated population.

Clearly, the ability to define alloreactive clones and detect expansions and reductions in these clones following transplant is highly dependent on the sample size of pretransplant unstimulated $\mathrm{T}$ cell populations. As any sample identifies only a fraction of the total TCR repertoire, these numerical considerations are enormously important for the interpretation of data. With the depth of sequencing available using the "deep sequencing" platform by Adaptive Biotechnologies, we were able to demonstrate a high level of overlap of clones identified as responsive to a given donor using different pools of responder cells drawn from the same volunteer up to a year apart (91). This observation is consistent with our observation (91) and those of others $(77,92,104)$ that the circulating TCR repertoire is quite stable over time in a given individual, but also indicates that the clones we identify as alloreactive from a blood draw are sufficiently abundant to have a high probability of being detected within another similar-sized pool of $\mathrm{T}$ cells from a later blood draw. This technical aspect is critical for interpretation of serial samples assessing the fate of alloreactive clones over time. Obtaining sufficient sensitivity for the detection of low-frequency clones in unstimulated $\mathrm{T}$ cell populations is especially important for drawing statistically valid conclusions regarding loss of alloreactive $\mathrm{T}$ cell clones following transplant. In contrast, expansions of alloreactive clones can be detected in small samples such as biopsy specimens (98); however, the ability to identify these expansions and hence the sensitivity of the assay is highly dependent on having a robust CFSE-low and unstimulated sequence set before transplant from which to identify a maximal number of alloreactive clones. Additionally, the integral role of bioinformatics expertise in these analyses must be emphasized.

The TCR sequencing approach we have developed focuses on tracking alloreactive clones defined before transplantation (91). While this approach has the advantage of not depending on post-transplant functional assays in the setting of immu- 
nosuppression and possible anergy-inducing conditions that may affect in vitro cell proliferation, it has the disadvantage of failing to identify $\mathrm{T}$ cells that develop de novo after transplant, which may be donor-reactive. This method could also miss the impact of cross-reactive $\mathrm{T}$ cell clones that are present before transplant at too low a frequency for detection in CFSE-MLR, and that expand in response to post-transplant infections (105). It will therefore be important to develop strategies for identifying post-transplant alloreactive clones; similarly, combining sequencing results with additional functional assays and/or focusing on phenotypic subpopulations such as Tregs will provide important insight into the functional nature of the alloreactive $\mathrm{T}$ cell population. However, our observation that most alloreactive clones are present at very low frequency in the unstimulated repertoire argues against the notion that most alloreactivity reflects the activity of pathogen-reactive memory $\mathrm{T}$ cells that cross-react to alloantigens. While it is possible that by excluding pathogen-reactive clones that are dominant in the $\mathrm{T}$ cell repertoire with our fold-expansion criterion, we miss the contribution of such clones to the alloresponse, these clones are, by definition in our assay, relatively weak in their cross-reaction to the donor. Rarity of such cross-reactions could explain the ability to diminish GVHD by depleting naive T cells and transferring only memory T cells in HSCT recipients (30). However, the depletion of "naive" cells in the cited study was based only on CD45RA expression, and therefore also included depletion of the memory subset known as T effector memory RA+ (TEM$\mathrm{RA}$ ) cells and of $\mathrm{T}$ memory stem cells. It is also possible that prominence of "memory" $\mathrm{T}$ cells in allograft rejection studies $(106,107)$ may in fact reflect $T$ cells that have adopted the memory phenotype as a result of lymphopenia-associated expansion (74), which is in fact driven by donor alloantigens and therefore is biased toward the expansion of formerly naive donor-reactive $\mathrm{T}$ cells. Future studies involving assignment of alloreactive $\mathrm{T}$ cells before transplant to sorted naive, memory, and Treg subsets separated by cell sorting will help to elucidate the role of each cell type following transplantation.

Another limitation of the MLR/TCR sequencing-based alloreactivity assay is that it focuses primarily on the direct pathway of allorecognition. While we typically start with whole peripheral blood mononuclear cell responder populations in the CFSE-MLR in order to permit indirect alloresponses, specific modifications of the assay are needed to explicitly distinguish indirectly from directly alloreactive TCRs with this approach. While the focus of our assay only on the $\beta$ chain of the TCR may be seen as another limitation, single-cell approaches needed to identify $\alpha \beta$ TCR pairs currently do not allow the high-throughput work flow needed for the types of tracking studies we perform, though future technological advances (108) and computational approaches (109) to $\alpha \beta$ TCR pairing may permit such analyses. While strong HLA mismatches are associated with vigorous CFSE-MLR results, the extent of proliferation is much less in the nearly or fully HLA-matched setting (refs. 90, 110, and our unpublished observations), despite the presence of clinically important alloantigens. Development of refined techniques for the closely HLA-matched setting will therefore also be important, particularly for HSCT.

\section{Clinical implications and future directions}

As TCR sequencing assays for tracking alloreactive T cell populations mature, potential clinical applications abound. While the tolerant CKBMT cohort described by Morris et al. (91) is a unique population and comparable tolerant transplant populations are limited, there are many other potential biomarker applications for tracking donor-specific TCRs in transplant patients. Tracking of these clones in the circulation, biopsies, and urine specimens may ultimately be used to aid in decisions to increase or de-escalate immunosuppressive therapy, to diagnose or even predict rejection, and to monitor responses to antirejection therapy. Perhaps most tantalizing is the potential to identify the rare operationally tolerant kidney transplant recipient in whom immunosuppression could be safely withdrawn or to predict success or failure of structured immunosuppression weaning in liver transplant recipients. The need for biomarkers for this purpose is acute, and alloreactive TCR tracking is unique in its specificity for donor antigen reactivity; thus, further investigation of the potential of this approach is warranted. Defining the impact of particular immunosuppressive regimens on alloreactive clones may help tailor immunosuppression and provide invaluable information about how different immunomodulating agents directly affect donor-specific clones.

TCR sequencing approaches could increase our understanding of the role of HLA disparity in determining the size, diversity, quality, and ontogeny of this uniquely large repertoire. A critical evaluation of the role of pathogen-specific TCRs that are cross-reactive to allo-MHC is needed to determine the extent to which such cross-reactivity drives the alloresponse (66). As the importance of TCR sequencing approaches emerges, the utility and accessibility of analytic tools will simultaneously expand, which is an integral component of accurately understanding the vast amount of data high-throughput techniques provide. Moreover, as the throughput of single-cell transcriptional analysis improves, it should ultimately be possible to carry out single-cell high-throughput TCR sequencing in combination with transcriptional analysis, thereby defining function for TCRs identified by paired $\alpha$ and $\beta$ chains.

\section{Conclusions}

Alloreactive $\mathrm{T}$ cells play a central role in transplantation: they are key mediators of tolerance, rejection, and GVHD. Questions remain about the role of various allorecognition pathways in allograft rejection in vivo and about the mechanisms by which naive and memory $\mathrm{T}$ cells may become alloreactive. Some of the many fundamental questions that remain include the relative contributions of naive and memory cells to rejection and GVHD, how HLA disparity influences the diversity of the alloresponse, and how different clinical protocols and pharmacologic agents may directly target each of these parameters. Though great strides have been made in characterizing this large and diverse population, the use of high-throughput TCR sequencing opens a new window for investigating many of these questions. Refinements in functional assays to identify specific alloreactive $\mathrm{T}$ cell populations along with improvements in sequencing technologies will further advance our understanding of the mechanisms of alloreactivity, with important clinical implications. Carefully designed clinical studies to investigate the diagnostic and predictive value of allore- 
active TCR expansion and contraction in the systemic circulation, the graft, and the urine could ultimately provide refined noninvasive diagnostic capacity and enhance our ability to personalize immunosuppressive regimens.

\section{Acknowledgments}

We thank TeShima Brennen for expert assistance with manuscript preparation. The work was supported in part by NIH grants P01
AI106697 and Immune Tolerance Network contract N01 AI15416. SD was supported by an HONORS award from the American Society of Hematology. MS is supported by grants from the NIH.

Address correspondence to: Megan Sykes, Columbia Center for Translational Immunology, Department of Medicine, Columbia University, 650 West 168th Street, BB 1512, New York, New York 10032, USA. Phone: 212.304.5696; Email:megan.sykes@columbia.edu.
1. Ford WL, Atkins RC. The proportion of lymphocytes capable of recognizing strong transplantation antigens in vivo. Adv Exp Med Biol. 1973;29(0):255-262.

2. Wilson DB. Quantitative studies on the mixed lymphocyte interaction in rats. J Exp Med. 1967;126(4):625-654.

3. Wilson DB, Silvers WK, Nowell PC. Quantitative studies on the mixed lymphocyte interaction in rats. J Exp Med. 1967;126(4):655-665.

4. Wilson DB, Blyth JL, Nowell PC. Quantitative studies on the mixed lymphocyte interaction in rats. 3. Kinetics of the response. J Exp Med. 1968;128(5):1157-1181.

5. Lindahl KF, Wilson DB. Histocompatibility antigen-activated cytotoxic T lymphocytes. II. Estimates of the frequency and specificity of precursors. J Exp Med. 1977;145(3):508-522.

6. Suchin EJ, Langmuir PB, Palmer E, Sayegh MH, Wells AD, Turka LA. Quantifying the frequency of alloreactive T cells in vivo: new answers to an old question. J Immunol. 2001;166(2):973-981.

7. Noorchashm $\mathrm{H}$, et al. A direct method for the calculation of alloreactive CD $4^{+} \mathrm{T}$ cell precursor frequency. Transplantation. 1999;67(9):1281-1284.

8. Nisbet NW, Simonsen M, Zaleski M. The frequency of antigen-sensitive cells in tissue transplantation. J Exp Med. 1969;129(3):459-467.

9. Sharrock CE, Man S, Wanachiwanawin W, Batchelor JR. Analysis of the alloreactive T cell repertoire in man. I. Differences in precursor frequency for cytotoxic $\mathrm{T}$ cell responses against allogeneic MHC molecules in unrelated individuals. Transplantation. 1987;43(5):699-703.

10. van Oers MH, Pinkster J, Zeijlemaker WP. Quantification of antigen-reactive cells among human $\mathrm{T}$ lymphocytes. Eur J Immunol. 1978;8(7):477-484.

11. Orosz CG, Adams PW, Ferguson RM. Frequency of human alloantigen-reactive T lymphocytes. Transplantation. 1987;43(5):718-724.

12. Sherman LA, Chattopadhyay S. The molecular basis of allorecognition. Annu Rev Immunol. 1993;11:385-402.

13. Ali JM, Bolton EM, Bradley JA, Pettigrew GJ. Allorecognition pathways in transplant rejection and tolerance. Transplantation. 2013;96(8):681-688.

14. Afzali B, Lechler RI, Hernandez-Fuentes MP. Allorecognition and the alloresponse: clinical implications. Tissue Antigens. 2007;69(6):545-556.

15. Pietra BA, Wiseman A, Bolwerk A, Rizeq M, Gill RG. CD4 T cell-mediated cardiac allograft rejection requires donor but not host MHC class II. J Clin Invest. 2000;106(8):1003-1010.

16. Lee RS, et al. Indirect recognition of allopeptides promotes the development of cardiac allograft vasculopathy. Proc Natl Acad Sci U S A.
2001;98(6):3276-3281.

17. Steele DJ, et al. Two levels of help for B cell alloantibody production. JExp Med. 1996;183(2):699-703.

18. Herrera OB, et al. A novel pathway of alloantigen presentation by dendritic cells. J Immunol. 2004;173(8):4828-4837.

19. Marino J, et al. Donor exosomes rather than passenger leukocytes initiate alloreactive T cell responses after transplantation. Sci Immunol. 2016;1(1):aaf8759.

20. Golshayan D, Wyss JC, Buckland M, Hernandez-Fuentes M, Lechler RI. Differential role of naive and memory CD4 T-cell subsets in primary alloresponses. Am J Transplant. 2010;10(8):1749-1759.

21. Macedo C, et al. Contribution of naive and memory T-cell populations to the human alloimmune response. Am J Transplant. 2009;9(9):2057-2066.

22. Forman D, et al. Viral abrogation of stem cell transplantation tolerance causes graft rejection and host death by different mechanisms. J Immunol. 2002;168(12):6047-6056.

23. Welsh RM, et al. Virus-induced abrogation of transplantation tolerance induced by donor-specific transfusion and anti-CD154 antibody. J Virol. 2000;74(5):2210-2218.

24. Williams MA, et al. Characterization of virus-mediated inhibition of mixed chimerism and allospecific tolerance. JImmunol. 2001;167(9):4987-4995.

25. Adams AB, et al. Heterologous immunity provides a potent barrier to transplantation tolerance. J Clin Invest. 2003;111(12):1887-1895

26. Brehm MA, Markees TG, Daniels KA, Greiner DL, Rossini AA, Welsh RM. Direct visualization of cross-reactive effector and memory allo-specific CD8 T cells generated in response to viral infections. JImmunol. 2003;170(8):4077-4086.

27. Zheng $\mathrm{H}$, et al. Effector memory $\mathrm{CD} 4^{+} \mathrm{T}$ cells mediate graft-versus-leukemia without inducing graft-versus-host disease. Blood. 2008;111(4):2476-2484.

28. Zhang P, Wu J, Deoliveira D, Chao NJ, Chen BJ. Allospecific CD4(+) effector memory T cells do not induce graft-versus-host disease in mice. Biol Blood Marrow Transplant. 2012;18(10):1488-1499.

29. Anderson BE, et al. Memory $\mathrm{CD}^{+} \mathrm{T}$ cells do not induce graft-versus-host disease. JClin Invest. 2003;112(1):101-108.

30. Bleakley M, et al. Outcomes of acute leukemia patients transplanted with naive $\mathrm{T}$ cell-depleted stem cell grafts. J Clin Invest. 2015;125(7):2677-2689.

31. Felix NJ, Allen PM. Specificity of T-cell alloreactivity. Nat Rev Immunol. 2007;7(12):942-953.

32. Sheldon S, Poulton K. HLA typing and its influence on organ transplantation. Methods Mol Biol.
2006;333:157-174.

33. Susal C, Opelz G. Current role of human leukocyte antigen matching in kidney transplantation. Curr Opin Organ Transplant. 2013;18(4):438-444.

34. Opelz G, Wujciak T, Dohler B, Scherer S, Mytilineos J. HLA compatibility and organ transplant survival. Rev Immunogenet. 1999;1(3):334-342.

35. Petersdorf EW. Optimal HLA matching in hematopoietic cell transplantation. Curr Opin Immunol. 2008;20(5):588-593.

36. Luznik L, et al. High-dose cyclophosphamide as single-agent, short-course prophylaxis of graft-versus-host disease. Blood. 2010;115(16):3224-3230.

37. Greco R, et al. Posttransplantation cyclophosphamide and sirolimus for prevention of GVHD after HLA-matched PBSC transplantation. Blood. 2016;128(11):1528-1531.

38. Pidala J, et al. Amino acid substitution at peptide-binding pockets of HLA class I molecules increases risk of severe acute GVHD and mortality. Blood. 2013;122(22):3651-3658.

39. Goulmy E, et al. Mismatches of minor histocompatibility antigens between HLA-identical donors and recipients and the development of graft-versus-host disease after bone marrow transplantation. NEngl J Med.1996;334(5):281-285.

40. Jerne NK. The somatic generation of immune recognition. Eur J Immunol. 1971;1(1):1-9.

41. Zerrahn J, Held W, Raulet DH. The MHC reactivity of the $\mathrm{T}$ cell repertoire prior to positive and negative selection. Cell. 1997;88(5):627-636.

42. Scott-Browne JP, White J, Kappler JW, Gapin L, Marrack P. Germline-encoded amino acids in the $\alpha \beta \mathrm{T}$-cell receptor control thymic selection. Nature. 2009;458(7241):1043-1046.

43. Feng D, Bond CJ, Ely LK, Maynard J, Garcia KC. Structural evidence for a germline-encoded T cell receptor-major histocompatibility complex interaction 'codon'. Nat Immunol. 2007;8(9):975-983.

44. Bevan MJ. High determinant density may explain the phenomenon of alloreactivity. Immunol Today. 1984;5(5):128-130.

45. Aosai F, et al. Different types of allospecific CTL clones identified by their ability to recognize peptide loading-defective target cells. Eur J Immunol. 1991;21(11):2767-2774.

46. Felix NJ, et al. Alloreactive T cells respond specifically to multiple distinct peptide-MHC complexes. Nat Immunol. 2007;8(4):388-397.

47. Morris GP, Ni PP, Allen PM. Alloreactivity is limited by the endogenous peptide repertoire. Proc Natl Acad Sci U S A. 2011;108(9):3695-3700.

48. Amir AL, et al. Allo-HLA-reactive T cells inducing graft-versus-host disease are single peptide specific. Blood. 2011;118(26):6733-6742.

49. Archbold JK, et al. Alloreactivity between 
disparate cognate and allogeneic pMHC-I complexes is the result of highly focused, peptide-dependent structural mimicry. J Biol Chem 2006;281(45):34324-34332.

50. Udaka K, Tsomides TJ, Eisen HN. A naturally occurring peptide recognized by alloreactive CD $8^{+}$ cytotoxic T lymphocytes in association with a class I MHC protein. Cell.1992;69(6):989-998.

51. Wucherpfennig KW, et al. Polyspecificity of T cell and B cell receptor recognition. Semin Immunol. 2007;19(4):216-224.

52. Felix NJ, et al. H2-DM $\alpha(-/-)$ mice show the importance of major histocompatibility complex-bound peptide in cardiac allograft rejection. JExp Med. 2000;192(1):31-40.

53. Chen J, Eisen HN, Kranz DM. A model T-cell receptor system for studying memory T-cell development. Microbes Infect. 2003;5(3):233-240.

54 . Mason D. A very high level of crossreactivity is an essential feature of the T-cell receptor. Immunol Today. 1998;19(9):395-404.

55. Reiser JB, et al. CDR3 loop flexibility contributes to the degeneracy of TCR recognition. Nat Immunol. 2003;4(3):241-247.

56. Colf LA, et al. How a single T cell receptor recognizes both self and foreign MHC. Cell. 2007;129(1):135-146.

57. Gras S, Kjer-Nielsen L, Chen Z, Rossjohn J, McCluskey J. The structural bases of direct T-cell allorecognition: implications for T-cell-mediated transplant rejection. Immunol Cell Biol. 2011;89(3):388-395.

58. Hennecke J, Wiley DC. Structure of a complex of the human alpha/beta T cell receptor (TCR) HA1.7, influenza hemagglutinin peptide, and major histocompatibility complex class II molecule, HLA-DR4 (DRA*0101 and DRB1*0401): insight into TCR cross-restriction and alloreactivity. J Exp Med. 2002;195(5):571-581.

59. Morris GP, Allen PM. Cutting edge: Highly alloreactive dual TCR T cells play a dominant role in graft-versus-host disease. JImmunol. 2009;182(11):6639-6643.

60. Morris GP, Uy GL, Donermeyer D, Dipersio JF, Allen PM. Dual receptor T cells mediate pathologic alloreactivity in patients with acute graft-versus-host disease. Sci Transl Med. 2013;5(188):188ra74

61. Schendel DJ, et al. Cytotoxic T lymphocytes show HLA-C-restricted recognition of EBV-bearing cells and allorecognition of HLA class I molecules presenting self-peptides. JImmunol. 1992;149(7):2406-2414.

62. Burrows SR, Khanna R, Burrows JM, Moss DJ. An alloresponse in humans is dominated by cytotoxic T lymphocytes (CTL) cross-reactive with a single Epstein-Barr virus CTL epitope: implications for graft-versus-host disease. J Exp Med. 1994;179(4):1155-1161.

63. Gaston JS, Rickinson AB, Epstein MA. Cross-reactivity of self-HLA-restricted Epstein-Barr virus-spe cific cytotoxic T lymphocytes for allo-HLA determinants. JExp Med.1983;158(6):1804-1821.

64. Koelle DM, Chen HB, McClurkan CM, Petersdorf EW. Herpes simplex virus type 2-specific CD8 cytotoxic T lymphocyte cross-reactivity against prevalent HLA class I alleles. Blood. 2002;99(10):3844-3847.
65. D’Orsogna LJ, Roelen DL, Doxiadis II, Claas FH. Alloreactivity from human viral specific memory T-cells. Transpl Immunol. 2010;23(4):149-155.

66. Amir AL, et al. Allo-HLA reactivity of virus-specific memory T cells is common. Blood. 2010;115(15):3146-3157.

67. Venturi V, et al. TCR $\beta$ eta-chain sharing in human $\mathrm{CD}^{+} \mathrm{T}$ cell responses to cytomegalovirus and EBV. J Immunol. 2008;181(11):7853-7862.

68. Trautmann L, et al. Selection of $\mathrm{T}$ cell clones expressing high-affinity public TCRs within Human cytomegalovirus-specific CD8 T cell responses. JImmunol. 2005;175(9):6123-6132.

69. Valkenburg SA, et al. Molecular basis for universal HLA-A ${ }^{*}$ 0201-restricted CD ${ }^{+}$T-cell immunity against influenza viruses. Proc Natl Acad Sci U S A. 2016;113(16):4440-4445

70. Dong L, Li P, Oenema T, McClurkan CL, Koelle DM. Public TCR use by herpes simplex virus-2-specific human CD8 CTLs. JImmunol. 2010;184(6):3063-3071.

71. Schenk AD, Nozaki T, Rabant M, Valujskikh A Fairchild RL. Donor-reactive CD8 memory T cells infiltrate cardiac allografts within 24 -h posttransplant in naive recipients. Am J Transplant. 2008;8(8):1652-1661.

72. Page AJ, Ford ML, Kirk AD. Memory T-cell-specific therapeutics in organ transplantation. Curr Opin Organ Transplant. 2009;14(6):643-649.

73. Yamada Y, et al. Overcoming memory T-cell responses for induction of delayed tolerance in nonhuman primates. Am J Transplant. 2012;12(2):330-340.

74. Onoe T, Kalscheuer H, Chittenden M, Zhao G, Yang YG, Sykes M. Homeostatic expansion and phenotypic conversion of human T cells depend on peripheral interactions with APCs. JImmunol. 2010;184(12):6756-6765.

75. Davis MM, Bjorkman PJ. T-cell antigen receptor genes and T-cell recognition. Nature. 1988;334(6181):395-402.

76. Yousfi Monod M, Giudicelli V, Chaume D, Lefranc MP. IMGT/JunctionAnalysis: the first tool for the analysis of the immunoglobulin and $\mathrm{T}$ cell receptor complex V-J and V-D-J JUNCTIONs. Bioinformatics. 2004;20(suppl 1):i379-i385.

77. Robins HS, et al. Comprehensive assessment of $\mathrm{T}$-cell receptor $\beta$-chain diversity in $\alpha \beta$ T cells. Blood. 2009;114(19):4099-4107.

78. Parham P. The immune system. 4th ed. New York, New York, USA: Garland Science; 2014.

79. Sherman LA. Dissection of the B10. JExp Med. 1980;151(6):1386-1397.

80. Bill J, et al. Molecular genetic analysis of 178 I-Abm12-reactive T cells. J Exp Med. 1989;169(1):115-133.

81. Sebille F, et al. Direct recognition of foreign MHC determinants by naive $\mathrm{T}$ cells mobilizes specific $\mathrm{V} \beta$ families without skewing of the complementarity-determining region 3 length distribution. Jimmunol. 2001;167(6):3082-3088.

82. Jeras $M$. The role of in vitro alloreactive T-cell functional tests in the selection of HLA matched and mismatched haematopoietic stem cell donors. Transpl Immunol. 2002;10(2-3):205-214.

83. Bain B, Vas M, Lowenstein L. Reaction between leukocytes in mixed peripheral blood cultures. Federation Proceedings. 1963;22:428.
84. Bach F, Hirschhorn K. Lymphocyte interaction: a potential histocompatibility test in vitro. Science. 1964;143(3608):813-814.

85. Parish CR. Fluorescent dyes for lymphocyte migration and proliferation studies. Immunol Cell Biol. 1999;77(6):499-508.

86. Lyons AB, Parish CR. Determination of lymphocyte division by flow cytometry. J Immunol Methods. 1994;171(1):131-137.

87. Heeger PS, et al. Pretransplant frequency of donor-specific, IFN- $\gamma$-producing lymphocytes is a manifestation of immunologic memory and correlates with the risk of posttransplant rejection episodes. JImmunol. 1999;163(4):2267-2275.

88. Augustine JJ, Poggio ED, Heeger PS, Hricik DE. Preferential benefit of antibody induction therapy in kidney recipients with high pretransplant frequencies of donor-reactive interferon-gamma enzyme-linked immunosorbent spots. Transplantation. 2008;86(4):529-534.

89. Moris A, Teichgräber V, Gauthier L, Bühring HJ, Rammensee HG. Cutting edge: characterization of allorestricted and peptide-selective alloreactive T cells using HLA-tetramer selection. Jimmunol. 2001;166(8):4818-4821.

90. Michálek J, Collins RH, Hill BJ, Brenchley JM, Douek DC. Identification and monitoring of graftversus-host specific T-cell clone in stem cell transplantation. Lancet. 2003;361(9364):1183-1185.

91. Morris H, et al. Tracking donor-reactive T cells: Evidence for clonal deletion in tolerant kidney transplant patients. Sci Transl Med. 2015;7(272):272ra210.

92. Muraro PA, et al. T cell repertoire following autologous stem cell transplantation for multiple sclerosis. JClin Invest. 2014;124(3):1168-1172.

93. Miqueu P, et al. Analysis of the peripheral T-cell repertoire in kidney transplant patients. Eur J Immunol. 2010;40(11):3280-3290.

94. Tan JT, et al. IL-7 is critical for homeostatic proliferation and survival of naive T cells. Proc Nat Acad Sci U S A. 2001;98(15):8732-8737.

95. Kieper WC, et al. Recent immune status determines the source of antigens that drive homeostatic T cell expansion. JImmunol. 2005;174(6):3158-3163.

96. Sprangers B, et al. Origin of enriched regulatory t cells in patients receiving combined kidney/bone marrow transplantation to induce transplantation tolerance [published online ahead of print March 1,2017] Am JTransplant.https://doi.org/10.1111/ajt.14251.

97. Halloran PF. T cell-mediated rejection of kidney transplants: a personal viewpoint. Am J Transplant. 2010;10(5):1126-1134.

98. Zuber J, et al. Bidirectional intragraft alloreactivity drives the repopulation of human intestinal allografts and correlates with clinical outcome. Sci Immunol. 2016;1(4):eaah3732.

99. Zuber J, et al. Macrochimerism in Intestinal Transplantation: Association With Lower Rejection Rates and Multivisceral Transplants, Without GVHD. Am J Transplant. 2015;15(10):2691-2703.

100.Emerson RO, Mathew JM, Konieczna IM, Robins HS, Leventhal JR. Defining the alloreactive T cell repertoire using high-throughput sequencing of mixed lymphocyte reaction culture. PLoS One. 2014;9(11):e111943.

101.Frentsch M, et al. Direct access to CD4 ${ }^{+} \mathrm{T}$ cells 
specific for defined antigens according to CD154 expression. Nat Med. 2005;11(10):1118-1124.

102.Ashokkumar C, et al. Allospecific CD154 ${ }^{+} \mathrm{T}$ cells associate with rejection risk after pediatric liver transplantation. Am J Transplant. 2009;9(1):179-191.

103. Dziubianau $M$, et al. TCR repertoire analysis by next generation sequencing allows complex differential diagnosis of $\mathrm{T}$ cell-related pathology. Am J Transplant. 2013;13(11):2842-2854.

104. Robins HS, et al. Overlap and effective size of the human $\mathrm{CD} 8^{+} \mathrm{T}$ cell receptor repertoire. Sci Transl
Med. 2010;2(47):47ra64.

105. DeWolf S, Morris H, Shen Y, Sykes M. Author response to comment on "Tracking donor-reactive T cells: Evidence for clonal deletion in tolerant kidney transplant patients". Sci Transl Med. 2015;7(297):297lr1.

106.Kawai T, et al. HLA-mismatched renal transplantation without maintenance immunosuppression. N Engl J Med. 2008;358(4):353-361.

107. Andreola G, et al. Mechanisms of donor-specific tolerance in recipients of haploidentical combined bone marrow/kidney transplantation. Am J
Transplant. 2011;11(6):1236-1247.

108.Zheng GX, et al. Massively parallel digital transcriptional profiling of single cells. Nat Commun. 2017;8:14049.

109. Howie B, et al. High-throughput pairing of T cell receptor $\alpha$ and $\beta$ sequences. Sci Transl Med. 2015;7(301):301ra131.

110.Scheinberg P, Price DA, Ambrozak DR, Barrett AJ, Douek DC. Alloreactive T cell clonotype recruitment in a mixed lymphocyte reaction: implications for graft engineering. Exp Hematol. 2006;34(6):788-795. 